\title{
Adult neurogenesis in natural populations
}

\author{
R. Boonstra, L. Galea, S. Matthews, and J.M. Wojtowicz
}

\begin{abstract}
The dogma that the adult brain produces no new neurons has been overturned, but the critics are still asking, so what? Is adult neurogenesis a biologically relevant phenomenon, or is it perhaps harmful because it disrupts the existing neuronal circuitry? Considering that the phenomenon is evolutionarily conserved in all mammalian species examined to date and that its relevance has been well documented in non-mammalian species, it seems self-evident that neurogenesis in adult mammals must have a role. In birds, it has been established that neurogenesis varies dramatically with seasonal changes in song production. In chickadees, the learning behaviour related to finding stored food is also correlated with seasonal adult neurogenesis. Such studies are still nonexistent in mammals, but the related evidence suggests that neurogenesis does vary seasonally in hamsters and shows sexual differences in meadow voles. To promote studies on natural populations asking fundamental questions of the purpose and function of neurogenesis, we organized a Workshop on "Hippocampal Neurogenesis in Natural Populations" in Toronto in May 2000. The Workshop highlighted recent discoveries in neurogenesis from the lab, and focused on its functional consequences. The consensus at the Workshop was that demonstration of a role for neurogenesis in natural behaviours will ultimately be essential if we are to understand the purpose and function of neurogenesis in humans.
\end{abstract}

Key words: neurogenesis, hippocampus, dentate gyrus, learning, memory, wild population.

Résumé : Le dogme selon lequel le cerveau adulte ne produit pas de nouveaux neurones ne tient plus, mais les critiques continuent à dire, et alors ? La neurogenèse chez l'adulte est-elle un phénomène biologiquement pertinent, ou peut-elle être dommageable parce qu'elle perturbe le circuit neuronal existant ? Compte tenu du fait que le phénomène a été bien conservé au cours de l'évolution chez tous les mammifères examinés à ce jour et que sa pertinence a été bien documentée chez d'autres espèces, il semble aller de soi que la neurogenèse doit avoir un rôle chez les mammifères adultes. Chez les oiseaux, il est bien établi que la neurogenèse varie considérablement avec les variations saisonnières de la production de chants. Chez les mésanges, le comportement d'apprentissage lié à la découverte de la nourriture entreposée est aussi corrélé avec une neurogenèse saisonnière chez l'adulte. Il n'existe toujours pas d'études de ce genre chez les mammifères, mais les données connexes donnent à penser que la neurogenèse varie effectivement de façon saisonnière et montre des différences sexuelles chez les campagnols des champs et dans les variations du rythme circadien chez les hamsters. Pour encourager les études sur les populations naturelles abordant les questions fondamentales du but et de la fonction de la neurogenèse, nous avons organisé un colloque intitulé " Hippocampal Neurogenesis in Natural Populations ", à Toronto, au mois de mai 2000. Le Colloque a donné un aperçu des récentes découvertes sur la neurogenèse en laboratoire et mis l'accent sur ses conséquences fonctionnelles.. Selon le consensus établi lors du Colloque, il faudra nécessairement démontrer le rôle de la neurogenèse dans les comportements naturels si nous voulons comprendre le but et la fonction de la neurogenèse chez les humains.

Mots clés : neurogenèse, hippocampe, corps godronné, apprentissage, mémoire, population sauvage.

[Traduit par la Rédaction]

\section{Introduction}

The Workshop took place in Toronto, Canada on May 1113, 2000. Thirty scientists and 10 postdoctoral fellows and students from Canada, Germany, Japan, Sweden, Switzerland, and the U.S.A. attended the meeting. The scientific agenda consisted of nine symposium style presentations, one ad hoc session for short presentations (allowing all other participants to present their research to the group), and discussion sessions in four groups. The social events and meals maximized

Received September 22, 2000. Published on the NRC Research Press Web site on March 26, 2001.

R. Boonstra. Division of Life Sciences, University of Toronto at Scarborough.

L. Galea. Department of Psychology, University of British Columbia.

S. Matthews. Department of Physiology, University of Toronto.

J.M. Wojtowicz. ${ }^{1}$ Department of Physiology, University of Toronto, Medical Sciences Building, Toronto, ON M5S 1A8, Canada.

${ }^{1}$ Corresponding author (e-mail: martin.wojtowicz@utoronto.ca). 
discussion and interaction in the friendly, cottage style setting of the conference center.

\section{Overview and rationale}

Conventional wisdom has it that no new neurons form after birth. However, recent research has discovered that substantial neurogenesis occurs in the dentate gyrus of the hippocamus throughout life in all mammals, including humans. The hippocampus is a region of the brain that is central to learning, memory, and spatial ability. The major enigma in research on neurogenesis is why it occurs. To date, virtually all studies on mammals have been 'natureblind'. Studies have been unable to determine how the presence of this trait promotes survival and reproductive fitness in the natural world. In contrast, elegant research on birds (Barnea and Nottebohm 1996) implicates a fundamental role for hippocampal neurogenesis in bird song and food storage recovery. In mammals, laboratory studies on mice and rats indicate that survival of newly-produced neurons in the adult hippocampus is augmented by learning and that cell proliferation is inhibited by stress. However, while laboratory studies have been extremely valuable in increasing our understanding of the basic mechanisms of neurogenesis, it is difficult to extrapolate these findings to the natural world (of which humans are a part), and thus to determine how neurogenesis benefits both animals and humans in the struggle for survival.

It is now clear that the process of adult neurogenesis has been conserved in mammalian evolution. It has been demonstrated in a variety of species from mice to macaques, and includes humans (Eriksson et al. 1998). A variety of environmental factors are known to modify the rate of new neuron production. For example, environmental stress is a potent suppressor of neurogenesis (Tanapat et al. 1998). In contrast, hippocampal-dependent learning increases the survival of new neurons (Gould et al. 1999a), while exposure to an enriched environment (which is probably more akin to natural living conditions) also increases cell survival of newly-produced neurons and increases hippocampaldependent learning (van Praag et al. 1999). Obviously, exposure to stress and learning are highly integrated and experiments using natural populations can establish whether this relationship has significant consequences on animal fitness. Reduced hippocampal size and learning performance due to stress or exposure to chronically elevated adrenal steroids have been reported in aging humans and individuals suffering from Cushing's syndrome, depression, and posttraumatic stress disorder (Lupien et al. 1998). In contrast, an enlargement of posterior hippocampi was found in taxi drivers exposed to extensive navigational training (Maguire et al. 2000). Further understanding of the extent and function of adult neurogenesis will be necessary to determine whether these changes in hippocampal size and neurogenesis are in fact related.

Early brain development is attenuated by stress hormones (Matthews 2000). Therefore, hippocampal development and performance prenatally and postnatally are profoundly attenuated by stress. Furthermore, schizophrenia and depressive illness have been associated with stress and abnormal levels of stress hormones. Given this information, the crucial link between stress and the hippocampal performance is likely to be neurogenesis.

\section{Workshop objectives}

There are three justifications for the study of neurogenesis in natural populations:

(i) Biological realism: How do mammals in their natural environment benefit from adult neurogenesis?

(ii) Biological regulation: Do the processes known to modify neurogenesis in the laboratory operate in the same way in the natural environment?

(iii) Biological diversity: Animals in nature have evolved a diversity of mechanisms to solve the challenges of survival and reproduction. Knowledge of this diversity as it relates to neurogenesis should give us fundamental insight into how neurogenesis may be modified to meet the challenges of existence.

Ultimately, we wish to extrapolate findings in neurogenesis of natural populations of mammals to humans and how various interventions may be applicable to humans. Thus, the objectives of the workshop were: $(i)$ to focus on neurogenesis in the mammalian brain of natural populations, to review the current status of the field, and (ii) to plan future directions and collaborations with the ultimate goal of applying these findings in the human health arena.

\section{Specific questions and challenges discussed}

Six questions were posed to serve as a foundation for discussing the utility of natural populations:

(i) Can studies of natural populations in normal environmental conditions provide fundamental insights into the role and function of neurogenesis?

(ii) Can studies of natural populations be undertaken with sufficient rigor to provide robust data on neurogenesis given that wild animal studies have identified profound long-term changes in endocrine function during sustained exposure to environmental stress?

(iii) Is adult neurogenesis a prominent feature in wild animals and does it vary as a function of life history?

(iv) Are rates of adult neurogenesis constant with age or do they vary with life stages (pre-reproductive, reproductive, and post-reproductive)?

(v) Can stress during development (either prenatally or postnatally), which is known to program HPA axis function, alter parameters of neurogenesis?

(vi) Do natural stressors alter the rate of neurogenesis and ageing and does this have consequences for survival, ageing, and reproduction?

\section{Summary of symposium}

G. Kuhn (Regensburg, Germany) set the stage for the workshop by reviewing adult mammalian neurogenesis in both the olfactory bulb (approximately 60000 new cells/4 days) and the dentate gyrus (2000 cells/4 days). He elegantly portrayed a field in which knowledge appears to be doubling every 3 years and where the complexity of modifying factors (growth factors, serotonin, FGF-2, and EGF) is 
rapidly being appreciated. Apoptosis claims a large proportion of nascent cells. L. Galea (Vancouver, Canada) discussed what is known of neurogenesis in natural populations, indicating how natural stress simulators (i.e., predator odour) inhibit cell proliferation in the adult rat and how sex hormones, such as estrogen, initially enhance and subsequently suppress cell proliferation. H.-P. Lipp (Zurich, Switzerland) described his fascinating research on natural populations of house mice in enclosures in Russia. Variation in the mossy fiber projections formed by axons of neurons in the dentate gyrus is caused by variation in development (both early and adult) and is clearly influenced by natural selection. Ultimately this variation causes behavioral differences, which influence population dynamics. The link between the mossy fiber field and adult neurogenesis is currently being explored. M. Wojtowicz (Toronto, Canada) provided a graphical model of cell birth and death in the dentate gyrus. He also presented new research showing how these putative young neurons can produce long-term potentiation (a proposed cellular mechanism of learning in the hippocampus (Wang et al. 2000). This would imply that new memory pathways may route themselves through young, but not old, neurons. G. Kempermann (Regensburg, Germany) discussed the critical link between environment, learning, and the degree of neurogenesis. Environmental enrichment and activity can clearly stimulate neurogenesis and its purpose may be a direct function of novelty. His presentation raised some exciting new challenges. For example, do new neurons improve survival? Are they selective for the environment which increased the degree of neurogenesis or are these neurons multitasking?

Three talks had direct implications for human health. The landmark study of (Eriksson et al. 1998) clearly indicated that neurogenesis occurs in humans. They exploited a unique opportunity using a group of patients pre-injected with bromodeoxyuridine (BrdU) for the purpose of monitoring the growth of tumors in various parts of the body. Post mortem examination of their brains showed clear evidence of neurogenesis in several patients of advanced ages (60-70 years old). Eriksson (Goteborg, Sweden) then went on to discuss how the progenitor cell proliferation is regulated by insulin growth factor (IGF-I), selectively inducing neurogenesis. This was particularly exciting research which has direct implications for the therapeutic replacement of lost neurons in neurodegenerative diseases. Human stress hormone related changes in the hippocampal size imaged with MRI were presented by S. Lupien (Montreal, Canada). She convincingly showed a direct relationship between high levels of cortisol, memory impairment, and decline in hippocampal volume. With further progress of imaging techniques it should be possible to correlate such changes with the rates of neurogenesis. T. Seki (Tokyo, Japan) presented his pioneering studies of neurogenesis using antibodies to an immature form of polysialic acid-neural cell adhesion molecule (PSA-NCAM), a growth associated protein, present preferentially in new neurons in the dentate gyrus. The PSANCAM antibody has been successfully used to label postmortem and surgically removed brain material from normal and epileptic children (Mathern et al. 1999). Studies such as these will provide exciting further insights into the process of neurogenesis in humans.

\section{Challenges and future directions addressed in the discussion groups}

Four discussion groups provided the forum for intensive interactions and centered around four major themes:

(i) Cellular interactions in neurogenesis;

(ii) Neurogenesis and behavior;

(iii) Neurogenesis and the life cycle;

(iv) Neurogenesis in natural populations.

All groups highlighted a critical methodological issue still plaguing the field. BrdU represents a critical tool in measuring the rate of mitosis among the precursor cell population in the dentate gyrus. Yet, by its very nature, BrdU can be toxic to the dividing cells because it replaces endogenous thymidine. While no toxic effects of BrdU have been reported at the usual low doses $(50-100 \mathrm{mg} / \mathrm{kg}$ ) in the dentate gyrus, some developmental changes in the brain have been reported after long survival periods (Kolb et al. 1999). Thus, the discovery and validation of new non-toxic markers of mitosis are essential. Post-mitotic neurons can be identified with a combination of several antibody markers such as neuronal nuclear protein $(\mathrm{NeuN})$, collapsin response mediator protein 4 (CRMP-4; formerly called TOAD-64), polysialylated neural cell adhesion molecule (PSA-NCAM), and calbindin, yet their exact chronological ages cannot be determined with certainty. New and more accurate markers of cell age are needed. Another limitation with BrdU is that it requires pulse administration, usually via an intra-peritoneal injection. This is a potentially stressful procedure that is likely to inhibit the rate of neurogenesis through release of adrenal stress hormones. Better methods of BrdU administration are needed.

Another issue requiring urgent attention is the functionality of the new neurons. Although experiments did show that putative new neurons have different morphological and physiological characteristics (Wang et al. 2000), these studies also show that the young neurons are a small minority of the total cell population. It is presently not known if young neurons perform a significant role in the adult dentate gyrus. Behavioral analysis of the consequences of adult neurogenesis is crucial in establishing if new neurons are required for the animal survival. It was suggested that this issue could be resolved using the natural populations as experimental models, especially if the results are to be relevant to humans. Existing studies have already shown that an enriched environment and physical exercise (running) can enhance the rate of neurogenesis (van Praag et al. 1999). However, it is unclear how these observations might apply to natural populations living in environments that are presumably more enriched than the most complex environment simulated in the laboratory. Furthermore, stressful situations that exist in the natural habitat such as predation and competition for mates or territories cannot be recreated under laboratory conditions with laboratory-bred animals.

It was agreed that the studies of neurogenesis in natural populations are most worthwhile when performed in a rigorous fashion and if they tap into the achievements of the current research on laboratory animals. Thus, collaborative projects appear most promising if the researchers who are presently conducting laboratory studies actively seek out joint projects with ecologists and natural biologists. Two 
attendees of the workshop have extensive experience and the facilities for field work on natural populations. H.-P. Lipp has access to a colony of small mammals (house mice and voles) in Russia that can be monitored and manipulated in a sophisticated way with the use of telemetry. R. Boonstra works at a field station in the Yukon (Canada) where he can monitor wild populations of various species including small mammals, ground squirrels, and snowshoe hares. It should be possible for all those studying the significance of hippocampal neurogenesis to establish collaborative projects with researchers who have similar experience and facilities. It should also be possible to establish new collaborations with clinicial scientists who have access to human brain material.

Finally, it will be crucial to verify the existing results (Gould et al. 1999b) suggesting that adult neurogenesis occurs in other brain regions besides the dentate gyrus and the subventricular zone. If so, the clinical applications of the lessons learned from the dentate gyrus may have widespread significance for promoting regeneration in the injured or diseased brain.

In summary, the main objective of this workshop was to initiate new research collaborations and directions among the participants. The area of adult neurogenesis is of fundamental importance and will proceed down many novel and exciting avenues in the coming years. The functional significance of neurogenesis and how it promotes survival and fitness in natural populations (including humans) remains to be established. A key factor in advancing our understanding of the function and physiological significance of neurogenesis is in choosing appropriate experimental models and elucidating its role in the natural world.

\section{Key note presentations}

1. The regulation and functional significance of adult neurogenesis in natural populations of mammals. $\mathrm{R}$. Boonstra, Division of Life Sciences, University of Toronto at Scarborough, Toronto, ON M1C 1A4, Canada. Neurogenesis in the dentate gyrus is an evolutionary conserved trait in both birds and mammals. The major enigma in understanding neurogenesis in adults is why it occurs in the first place. What is its adaptive significance? R. Boonstra, a population ecologist who is shifting his focus to neuroendocrinology, laid out 3 reasons (given above) why the study of neurogenesis in natural populations of mammals will provide critical insight into this ubiquitous feature: biological realism, the regulation of neurogenesis in nature, and biological diversity. He then reviewed the key areas where neurogenesis may play a role: spatial memory, the effects of stress on survival and development, changes as a function of age or biological need, and reproduction and the differences between the sexes as a function of social system.

2. Regulation of neurogenesis in the adult mammalian nervous system. P.S. Eriksson, Institute of Clinical Neuroscience, Sahlgrenska University Hospital, Goteborg University, Goteborg, Sweden, 413 45. There is now evidence supporting the assertion that stem cells residing within the brain may act as a source for neurogenesis in restricted sites of the adult brain. In several species, including humans, the dentate granule cell layer of the hippocampus exhibits neurogenesis throughout adult life. The ability to regulate adult neurogenesis may be of significant therapeutic value as a mechanism for replacing lost neurons. Recently, we and other investigators have shown that an enriched environment increases neurogenesis in the hippocampus. Running was specifically identified as one positive regulator of neurogenesis. Interestingly, endogenous insulin growth factor I (IGF-I) increases after physical exercise. Insulin growth factor is a growth-promoting peptide that has been shown to have neurotrophic properties. Using bromodeoxyuridine (BrdU) labeling, we found a significant increase in neurogenesis after peripheral IGF-1 administration. Our results suggest that IGF-I may be an important physiological regulator of neurogenesis in the adult central nervous system.

3. Hormonal regulation of neurogenesis in adult brain. L.A.M. Galea, B. Ormerod, E. Falconer, and M. Holmes. Department of Psychology and Neuroscience Program, University of British Columbia, Vancouver, BC V6T 1Z4, Canada. The dentate gyrus is unique as it retains the ability to produce new neurons in adulthood. In both wild and laboratory populations of meadow voles, non-breeding females with low levels of endogenous estradiol had very high levels of cell proliferation in contrast with breeding females that had high levels of endogenous estradiol. Experimental evidence indicated that cell proliferation was dependent on estradiol. High levels of estradiol initially increased ( $4 \mathrm{~h}$ post-injection), but subsequently suppressed (48 $\mathrm{h}$ post-injection), cell proliferation in the dentate gyrus of adult female voles. In addition, exposure to predator odour suppressed cell proliferation in male rats $24 \mathrm{~h}$ after exposure, but the suppression was no longer evident after 1 week of continuous exposure, indicating either an upregulation in subsequent cell proliferation or an enhancement of cell survival. Thus, reproductive status, through the actions of estradiol, and exposure to stress affects cell proliferation and survival in the dentate gyrus of adult rodents.

4. Analysis of neurogenesis and programmed cell death in the adult brain. H.G. Kuhn, Dept. Neurology, University of Regensburg, D-93053 Regensburg, Germany. A review of adult mammalian neurogenesis in the olfactory bulb and the dentate gyrus emphasized that the generation of neuronal cells from endogenous stem cell populations is balanced by programmed cell death, which eliminates supernumerous precursor cells and mature neurons (Biebl et al. 2000. Analysis of neurogenesis and programmed cell death reveals a self renewing capacity in the adult rat brain. Neuroscience Lett. 291: 17-20). This opens two possibilities for increasing neuronal production, first by direct stimulation of stem cell proliferation and second by reducing the natural cell death of already existing neural progenitors and stem cells. It has been shown in several studies that growth factors such as FGF-2 and EGF are effective in increasing stem cell proliferation in vivo. Nevertheless, the fate of the stem cell progeny is not necessarily neuronal, since stimulation with EGF can lead to an increased production in glial cells rather than neurons. It remains to be shown whether a reduction of cell death in areas of neurogenesis by caspase-inhibitors actually leads to additional functionally active neurons.

5. Natural selection of the infrapyramidal mossy fiber projection in feralized mice and small wild mammals. H.-P. 
Lipp, Institute of Anatomy, Zurich, CH-8058, Zwitzerland. Genetic correlations between the size of the infrapyramidal mossy fiber tract (IP-MF) and hippocampal task performance in mice have been found, but their functional meaning remains obscure. We performed two ecological studies. In the first, a founder population of mice derived from strains with large or small IP-MF was released into large outdoor pens in Russia, and changes in the IP-MF were monitored over 4 years of outdoor life. Free-living mice underwent significant reductions of the IP-MF projection that remained associated with behavioral changes such as reduced exploration. In the second study we examined the relationship between the size of the IP-MF projection and habitat complexity. Species in complex environments show more extended IP-MF. This implies that the common denominator of such IP-MF variations is the degree to which animals can cope with distracting and (or) emotional stimuli. Since this ability changes also from youth to age, granule cells and their axons might provide a substrate by which genetic factors can preset the degree of behavioral reactivity, whereas adult neurogenesis might tune it according to individual experience.

6. The impact of increased stress hormones on memory function and hippocampal volume in a human elderly population. S. Lupien ${ }^{1,2}$, N.P.V. Nair ${ }^{1}$, S. Brière ${ }^{1}$, B.S. McEwen ${ }^{3}$, R.L. Hauger ${ }^{4}$, and M.J. Meaney ${ }^{1}$. ${ }^{1}$ Laboratory of Human Psychoneuroendocrine Research, Douglas Hospital Research Center, McGill University, 6875 Blvd. Lasalle, Montreal, Verdun, QC H4H 1R3, Canada. ${ }^{2}$ Montreal Geriatric Institute. ${ }^{3}$ Rockefeller University. ${ }^{4}$ University of California, San Diego. Perhaps the most prominent feature of human aging is the variability in the decline of intellectual processes. The main objective of our research program is to understand the basis of these individual differences in neurological function amongst the elderly. We are examining whether individual differences in hypothalamic-pituitary-adrenal (HPA) activity contribute to the variability in neuropsychological function. We have shown that elderly subjects with significant long-term elevations of cortisol levels have marked impairment in the memory function known to be dependent upon hippocampal integrity. Moreover, these subjects also show a $14 \%$ decrease in hippocampal volume (measured by MRI) relative to elderly subjects with normal levels of cortisol. These results suggest that cumulative exposure to high cortisol levels in human aging can be a risk factor in the development of memory impairments and in hippocampal atrophy.

7. Role of PSA-NCAM in adult neurogenesis. T. Seki, Department of Anatomy, Juntndo University School of Medicine, 2-1-1 Hongo Bunkyo-ku, Tokyo 113, Japan. Several years ago I found that newly generated dentate granule cells in the adult hippocampus express polysialic acid -neural cell adhesion molecule (PSA-NCAM 1993, J. Neurosci. 13: 2351). This molecule is known to be essential in neuronal development. This implies that the PSA-NCAM is a useful marker for visualizing the developmental process of new neurons. A critical area in understanding the significance of neurogenesis is knowing how the developing granule cells form new neuronal circuits. I have demonstrated the following: $(i)$ the number of the newly generated granule cells decreases with age (NeuroReport, 6: 2479, 1995); (ii) the dendrites grow along residual radial glia (J. Comp. Neurol. 410: 503, 1999); (iii) the mossy fibers possess immature and mature boutons (J. Comp. Neurol. 410: 115, 1999); and (iv) PSA-NCAM contributes to mossy fiber bouton arrangement (J. Neurosci. 18: 3757, 1998).

8. Consequences of adult neurogenesis on synaptic plasticity in the dentate gyrus. J.M. Wojtowicz, N. Kee, J. Snyder, Department of Physiology, University of Toronto, Toronto, ON M5S 1A8, Canada. Approximately 10\% of all granule cells within adult dentate gyrus of the rat are labeled with immunohistochemical markers of immature neurons (PSA-NCAM and CRMP-4). These young neurons are continually born by mitotic divisions from a population of precursors located in the subgranular proliferative zone. Although regulation of this adult neurogenesis has been studied extensively, its consequences on hippocampal plasticity and functions have received little attention. We demonstrated that at least some of the young, adult-generated granule neurons are endowed with unique synaptic properties, including lack of inhibition and an enhanced ability to generate long-term potentiation, in contrast with mature neurons. Long-term potentiation, a putative cellular mechanism which exhibits features similar to some forms of learning, may be a mechanism in the proposed regulation of neurogenesis by neural activity. A more thorough understanding of the synaptic properties of new neurons should help in understanding the possible roles of these neurons in real life situations such as navigation in space, searching for food, and finding mates.

\section{Acknowledgements}

This project was funded by the Canadian Tri-Council Workshop / Networking Program, a Federal initiative to promote and support scientific interaction and development.

\section{References}

Barnea, A., and Nottebohm, F. 1996. Recruitment and replacement of hippocampal neurons in young and adult chickadees: an addition to the theory of hippocampal learning. Proc. Natl. Acad. Sci. U.S.A. 93: 714-718.

Eriksson, P.S., Perfilieva, E., Bjork-Eriksson, T., Alborn, A.M., Nordborg, C., Peterson, D.A., and Gage, F.H. 1998. Neurogenesis in the adult human hippocampus. Nat. Med. 4: 1313-1317.

Gould, E., Beylin, A., Tanapat, P., Reeves, A., and Shors, T.J. 1999a. Learning enhances adult neurogenesis in the hippocampal formation. Nat. Neurosci. 2: 260-265.

Gould, E., Reeves, A.J., Graziano, M.S., and Gross, C.G. $1999 b$. Neurogenesis in the neocortex of adult primates. Science (Washington, D.C.), 286: 548-552.

Kolb, B., Pedersen, B., Ballermann, M., Gibb, R., and Whishaw, I.Q. 1999. Embryonic and postnatal injections of bromodeoxyuridine produce age- dependent morphological and behavioral abnormalities. J. Neurosci. 19: 2337-2346.

Lupien, S.J., de Leon, M., de Santi, S., Convit, A., Tarshish, C., Nair, N.P., Thakur, M., McEwen, B.S., Hauger, R.L., and Meaney, M.J. 1998. Cortisol levels during human aging predict hippocampal atrophy and memory deficits. Nat. Neurosci. 1: 69-73.

Maguire, E.A., Gadian, D.G., Johnsrude, I.S., Good, C.D., Ashburner, J., Frackowiak, R.S., and Frith, C.D. 2000. 
Navigation-related structural change in the hippocampi of taxi drivers. Proc. Natl. Acad. Sci. U.S.A. 97: 4398-4403.

Mathern, G.W., Pretorius, J.K., Leite, J.P., and Adelson, P.D. 1999. Hippocampal neuropathology in children with severe epilepsy. In Childhood epilepsies and brain development. Edited by A. Nehlig, J. Motte, S.L. Moshe, and P. Plouin. John Libbey \& Company Ltd. pp. 171-185.

Matthews, S. 2000. Antenatal glucocorticoids and programming of the developing CNS. Ped. Res. 47: 291-300.

Tanapat, P., Galea, L.A.M., and Gould, E. 1998. Stress inhibits the proliferation of granule cell precursors in the developing dentate gyrus. Int. J. Dev. Neurosci. 16: 235-239.

van Praag, H., Kempermann, G., and Gage, F.H. 1999. Running increases cell proliferation and neurogenesis in the adult mouse dentate gyrus. Nat. Neurosci. 2: 266-270.

Wang, S., Scott, B.W., and Wojtowicz, J.M. 2000. Heterogenous properties of dentate gyrus granule neurons in the adult rat. J. Neurobiol. 42: 248-257. 